\title{
APPROXIMATE FIBRATIONS WITH NONFINITE FIBERS
}

\author{
STEVE FERRY ${ }^{1}$
}

\begin{abstract}
It is shown that every ANR fibration over $S^{1}$ with finitely dominated fiber is homotopy equivalent to an approximate fibration with compact ANR total space. This yields examples of approximate fibrations with arbitrary compact pointed FANR fibers.
\end{abstract}

1. Introduction. We start with some definitions. All spaces considered in this paper are separable and metric. If $Y$ is such a space and $\alpha$ is an open cover of $Y$, two maps $f, g: X \rightarrow Y$ are said to be $\alpha$-close if for each $x \in X$ there is a $U_{x} \in \alpha$ such that $f(x), g(x) \in U_{x}$.

If $\alpha$ is an open cover of $B$, then a map $p: E \rightarrow B$ is said to be an $\alpha$-fibration if for each $f: X \times I \rightarrow B$ and $F: X \times\{0\} \rightarrow E$ with $p \circ F=f \mid X \times\{0\}$ there is an extension $F^{\prime}: X \times I \rightarrow E$ of $F$ such that $p \circ F^{\prime}$ is $\alpha$-close to $f$. If $p$ is proper (i.e. inverse images of compacta are compact) and $p$ is an $\alpha$-fibration for each open cover $\alpha$ of $B, p$ is called an approximate fibration.

The geometric study of approximate fibrations was originated by Coram and Duvall $[$ C-D 1 , [C-D $]$ and has been continued by L. Husch $\left[\mathbf{H}_{1}\right],\left[\mathbf{H}_{2}\right], \mathbf{L}$. Mand [M], and R. Goad [G]. The point of view taken in these papers is to study approximate fibrations as generalized cell-like maps. The following results of Coram and Duvall motivated our work.

TheOREM [C-D ]. Let $E$ and $B$ be locally compact $A N R$ s and let $p: E \rightarrow B$ be an approximate fibration. Then

(i) a path $\omega: I \rightarrow B$ induces a shape equivalence from $p^{-1}(\omega(0))$ to $p^{-1}(\omega(1))$;

(ii) each fiber $p^{-1}(b)$ is a pointed fundamental ANR (pointed FANR).

An FANR is the shape analog of an ANR. The term is defined (along with the term "shape") in [B]. Our purpose in this paper is to prove a converse to part (ii), i.e., every pointed FANR is the fiber (up to shape) of some approximate fibration between compact ANRs.

The point of this is that a compact pointed FANR does not necessarily have the shape of a finite $\mathrm{CW}$ complex. The situation is outlined below. A space $K$ is said to dominate a space $L$ if there are maps $d: K \rightarrow L$ and $u$ : $L \rightarrow K$ such that $d \circ u$ is homotopic (equivalent) to id: $L \rightarrow L$. If the maps are shape maps, we speak of a shape domination. If they are ordinary maps,

Received by the editors July 1, 1976.

AMS (MOS) subject classifications (1970). Primary 54C10, 55F65, 57A20; Secondary 54C55, 57C05, 55F 15.

Key words and phrases. Approximate fibration, shape, finiteness obstruction.

${ }^{1}$ Supported by NSF grant MCS 76-06929. 
we speak of a homotopy domination. No shape theory appears in $\$ \S 2-4$ of this paper.

Facts about FANRs [S-G-H], [E-G], [Ch-F ] . (1) The following conditions are equivalent for compact spaces:

(i) $X$ is a pointed FANR.

(ii) $X$ is shape dominated by a finite $C W$ complex.

(iii) $X$ has the shape of a $C W$ complex.

(2) If $X$ is a connected pointed FANR, then there is an invariant $\sigma(X) \in$ $\tilde{K}_{0} Z \check{\pi}_{1} X$ which vanishes if and only if $X$ has the shape of a finite complex. All such obstructions are realized.

Here is the statement of our main theorem.

THEOREM 1.1. Let $\mathfrak{p}: \mathcal{E} \rightarrow S^{1}$ be a fibration of an $A N R$ over $S^{1}$. If the fiber $\mathfrak{p}^{-1}(1)$ is homotopy dominated by a finite complex, then there exist a compact $A N R E$, an approximate fibration $p: E \rightarrow S^{1}$, and a homotopy equivalence $g$ : $\mathcal{E} \rightarrow E$ such that the diagram

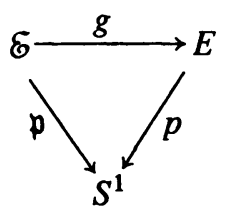

commutes up to homotopy.

The proof of Theorem 1.1 is the object of $\S 4$. Here is a theorem which helps to clarify the meaning of Theorem 1.1 .

THEOREM 1.2. If

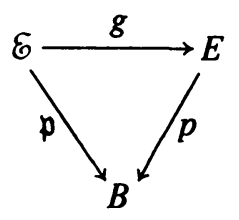

is a homotopy commuting diagram as above (with $S^{1}$ replaced by an $A N R B$ ), then $p^{-1}(b)$ is shape equivalent to $\mathfrak{p}^{-1}(b)$ for each $b \in B$.

Proof. Let $h$ be a homotopy inverse for $g$ and let $f: E \times I \rightarrow B$ be a homotopy from $\mathfrak{p} \circ h$ to $p$. Since $\mathfrak{p} \circ h=f \mid E \times\{0\}$, there is an extension of $h$ to a map $H: E \times I \rightarrow \mathcal{E}$ such that $\mathfrak{p} \circ H=f$. At the 1-level, $H_{1}: E \rightarrow \mathcal{E}$ is a homotopy equivalence such that $\mathfrak{p} \circ H_{1}=p$. We therefore have a map of long exact sequences 


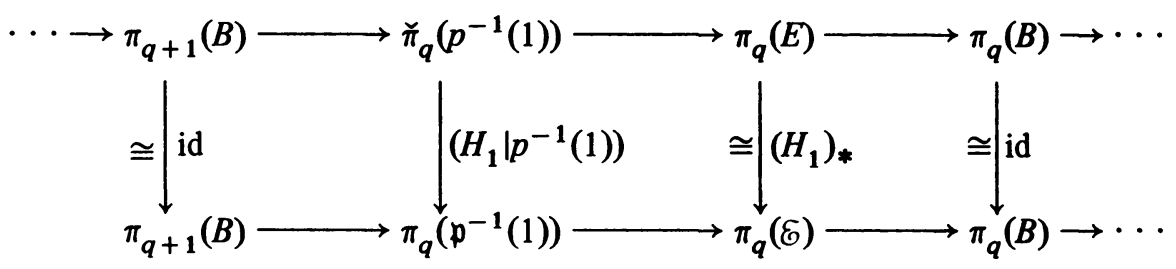

where the top sequence is the long exact sequence of an approximate fibration described in [C-D $]$. By the Five Lemma, $H_{1} \mid p^{-1}(1)$ induces isomorphisms of $\check{\pi}_{*}\left(p^{-1}(1)\right)$ and $\pi_{*}\left(p^{-1}(1)\right)$. Since $p^{-1}(1)$ has the shape of a CW complex, the Whitehead theorem implies that $H_{1} \mid p^{-1}(1)$ is a shape equivalence.

Here are some corollaries of Theorems 1.1 and 1.2.

COROllary 1.3. If $p: E \rightarrow B$ is an approximate fibration between connected $A N R s$, then $p^{-1}(b)$ is shape equivalent to the homotopy fiber of $p$.

COROLlaRy 1.4. If $L$ is a $C W$ complex which is homotopy dominated by a finite complex, then there is an approximate fibration $p: E \rightarrow S^{1}$ of compact $A N R s$ with fiber shape equivalent to $L$.

Proof. Apply Theorem 1.1 to the fibration $L \times S^{1} \rightarrow S^{1}$.

COROLlaRY 1.5. If an approximate fibration between ANRs is homotopic to a fibration, its fibers have the shapes of finite complexes.

Proof. If $p: E \rightarrow B$ and $p^{\prime}: E \rightarrow B$ are homotopic maps with $p$ an approximate fibration and $p^{\prime}$ a fibration, the diagram

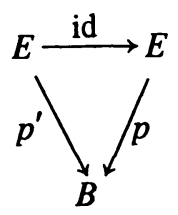

satisfies the hypotheses of Theorem 1.2. The fibers of $p^{\prime}$ are compact ANRs which, by $\left[\mathbf{W}_{1}\right]$, have the homotopy types of finite complexes.

2. Some facts about $Q$-manifolds. By the Hilbert cube we mean $Q=$ $\prod_{i=1}^{\infty}[-1,1]$. A Hilbert cube manifold is a separable metric manifold modelled on the Hilbert cube. A good basic reference on the topology of $Q$-manifolds is [Ch].

Definition 2.1. A closed subset $A$ of a $Q$-manifold $M$ is called a $Z$-set if for each open cover $\alpha$ of $M$ there is a map $f: M \rightarrow M-A$ such that for each $m \in M$ there is a $U_{m} \in \alpha$ with $m, f(m) \in U_{m}$ (i.e., $f$ is $\alpha$-close to id). An imbedding $f: X \rightarrow M$ is called a $Z$-imbedding if $f(X)$ is a $Z$-set in $M$. 
The basic result on $Z$-sets in $Q$-manifolds is the following theorem of Anderson and Chapman.

THEOREM 2.2 (Z-SET UNKNOTTING [A-Ch]). If $f, g: X \rightarrow M$ are proper homotopic Z-imbeddings, then there is a homeomorphism $h: M \rightarrow M$ such that $h \circ f=g$.

A proper surjection $f: X \rightarrow Y$ is said to be CE if each point-inverse is cell-like [L].

TheOREM 2.3. (R. D. EDWARDS [Ch]). If $X$ and $Y$ are locally compact ANRs and $f: X \rightarrow Y$ is a $C E$ map, then for each open cover $\beta$ of $Y \times Q$ there is a homeomorphism $\beta$-close to $f \times \mathrm{id}: X \times Q \rightarrow Y \times Q$. Moreover, $X \times Q$ and $Y \times Q$ are $Q$-manifolds.

Strictly speaking, none of our constructions requires Edwards' theorem-West's mapping cylinder theorem $\left[\mathbf{W}_{2}\right]$ would be entirely adequate. We will use Edwards' result to state general theorems for ANRs which we will use in the cases where the spaces involved are complexes or $Q$-manifolds. A case in point is the next proposition, which is a rewording of Theorem 21.2 of [Ch].

Proposition 2.4. If $X$ and $Y$ are locally compact $A N R$ s and $f: X \rightarrow Y$ is a homotopy equivalence, then $f \times \mathrm{id}: X \times Q \times[0,1) \rightarrow Y \times Q \times[0,1)$ is homotopic to a homeomorphism.

In view of the last two results, we introduce the following notation: if $f$ : $X \rightarrow Y$ is a map, then $\bar{X}$ will denote $X \times Q$, and $\bar{f}$ will denote $f \times$ id: $X \times Q \rightarrow Y \times Q$. Similarly, $\overline{\bar{f}}: \overline{\bar{X}} \rightarrow \overline{\bar{Y}}$ will denote $f \times$ id: $X \times Q \times[0,1)$ $\rightarrow Y \times Q \times[0,1)$.

We will also need the next result, which lies outside of $Q$-manifold theory. (Compare [Ch-F , Proposition 3.2].)

Proposition 2.5. Let $X$ and $Y$ be locally compact with $A$ a closed subset of $X$. If $f: X \rightarrow Y \times[0, \infty)$ is a map such that $f \mid A$ is proper, then $f$ is homotopic rel $A$ to a proper map $f_{1}: X \rightarrow Y \times[0, \infty)$.

Proof. Let $N$ be a closed neighborhood of $A$ such that $f \mid N$ is proper. Let $\rho$ : $X \rightarrow[0, \infty)$ be a proper map and let $\sigma: X \rightarrow[0,1]$ be a function which is identically 0 on $A$ and which is identically 1 outside of $N$. If $p_{Y}: Y \times[0, \infty)$ $\rightarrow Y$ and $p_{[0, \infty)}: Y \times[0, \infty) \rightarrow[0, \infty)$ are the projections, we define $f_{1}(x)=$ $\left(p_{Y} \circ f(x), p_{[0, \infty)} \circ f(x)+\sigma(x) \cdot \rho(x)\right)$. This clearly has the desired properties.

3. Facts about mapping cylinders. The purpose of this section is to establish some constructions for piecing together proper homotopy equivalences and 
homeomorphisms of mapping cylinders. These results are analogous to Lemmas 3.3-3.5 of [C].

DEFINITION 3.1. If

$$
X_{1} \stackrel{f_{1}}{\rightarrow} X_{2} \stackrel{f_{2}}{\rightarrow} \cdots \stackrel{f_{n-1}}{\rightarrow} X_{n}
$$

is a sequence of spaces and proper maps, we define the iterated mapping cylinder $M\left(f_{1}, \ldots, f_{n-1}\right)$ to be the space obtained from the disjoint union $\amalg_{i=1}^{n} X_{i} \times[0,1]$ by identifying $(x, 1) \in X_{i} \times I$ with $\left(f_{i}(x), 0\right) \in X_{i+1} \times I$. If $X_{1}=X_{n}$, we define the iterated mapping torus, $T\left(f_{1}, \ldots, f_{n-1}\right)$, to be the space obtained from $\mathbb{I}_{i=1}^{n-1} X_{i} \times[0,1]$ by identifying $(x, 1) \in X_{i} \times[0,1]$ with $\left(f_{i}(x) ; 0\right) \in X_{i+1} \times[0,1]$ for $i \leqslant n-2$ and by identifying $(x, 1) \in X_{n-1} \times$ $[0,1]$ with $\left(f_{n-1}(x), 0\right) \in X_{1} \times[0,1]$. Figure 1 illustrates $M\left(f_{1}, f_{2}\right)$ and $T\left(f_{1}, f_{2}\right)$.
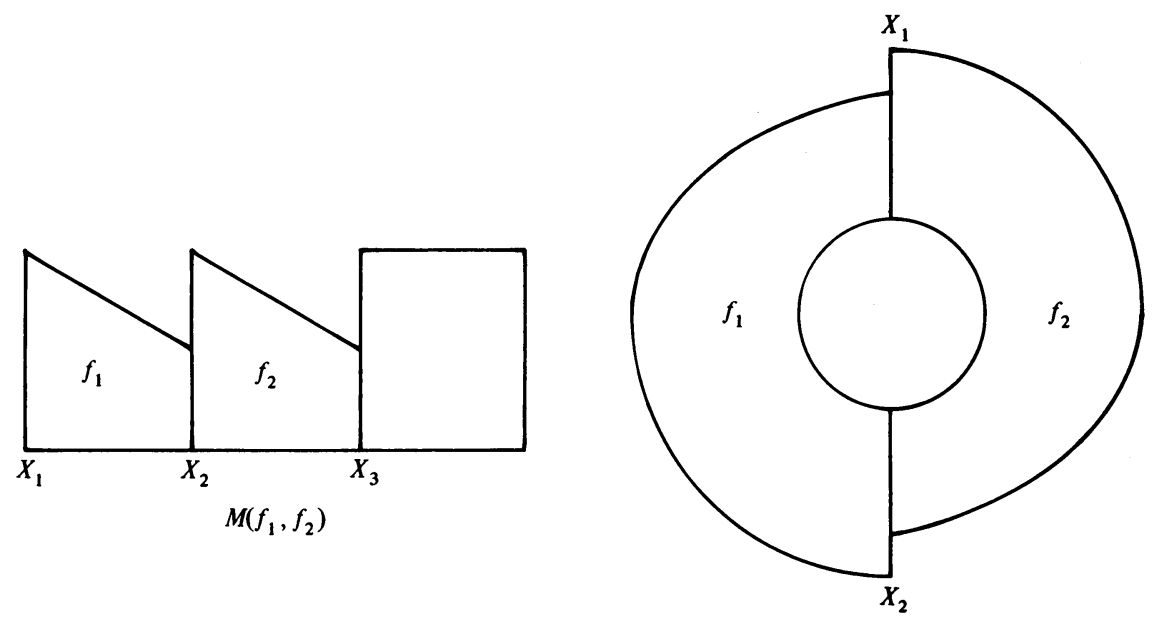

Figure 1

RemarK. (i) Note that $M\left(f_{1}\right)$ differs from the usual mapping cylinder in that a "pad" is added to the base. This makes the base a $Z$-set in $M\left(f_{1}\right)$.

(ii) There is a natural projection from $T\left(f_{1}, f_{2}, \ldots, f_{n}\right)$ to $S^{1}$. Each mapping cylinder projects to $(1 / n)$ th of the total circle.

Proposition 3.2. Let $X$ and $Y$ be locally compact ANRs and let $f, g: X \rightarrow Y$ be proper homotopic maps. Then:

(i) $M(f)$ and $M(g)$ are proper homotopy equivalent via homotopy equivalences $H$ and $G$ which are the identity on the ends $X \times\{0\}$ and $Y \times\{1\}$. Moreover, the homotopies from $G \circ H$ and $H \circ G$ to the identity are the identity on the ends at each level.

(ii) $M(\bar{f})$ and $M(\bar{g})$ are homeomorphic via a homeomorphism $h$ which is the identity on the ends $\bar{X} \times\{0\}$ and $\bar{Y} \times\{1\}$.

Proof. (ii) $\Rightarrow($ i). $\quad M(\bar{f})=M(f) \times Q$ and $M(\bar{g})=M(g) \times Q$, so we define 


$$
H: M(f) \stackrel{\times 0}{\rightarrow} M(f) \times Q \stackrel{h}{\rightarrow} M(g) \times Q \stackrel{\text { proj }}{\rightarrow} M(g)
$$

and

$$
G: M(g) \stackrel{\times 0}{\rightarrow} M(g) \times Q^{h^{-1}} \rightarrow M(f) \times Q \stackrel{\text { proj }}{\rightarrow} M(f) .
$$

These maps are the desired proper homotopy equivalences. Consider the composition:

$$
\begin{aligned}
M(f) & \stackrel{\times 0}{\rightarrow} M(f) \times Q \stackrel{h}{\rightarrow} M(g) \times Q \stackrel{\text { proj }}{\rightarrow} M(g) \\
& \stackrel{\times 0}{\rightarrow} M(g) \times Q \stackrel{h^{-1}}{\rightarrow} M(f) \times Q \stackrel{\text { proj }}{\rightarrow} M(f) .
\end{aligned}
$$

The homotopy from $(\times 0) \circ$ proj to id in the middle of the above diagram takes place in the $Q$-factor. Since $h$ and $h^{-1}$ are the identity on the ends, the composition provides a homotopy which is the identity on the ends at each stage.

(ii) By Theorem 2.3, the natural collapses $M(\bar{f}) \rightarrow \bar{Y}$ and $M(\bar{g}) \rightarrow \bar{Y}$ can be approximated by homeomorphisms $\gamma_{1}: M\left(\bar{f} \rightarrow \bar{Y}\right.$ and $\gamma_{2}: M(\bar{g}) \rightarrow \bar{Y}$. Since $\bar{f}$ and $\bar{g}$ are homotopic, $\gamma_{1} \mid(\bar{X} \times\{0\} \cup \bar{Y} \times\{1\})$ is homotopic to $\gamma_{2} \mid(\bar{X} \times\{0\} \cup \bar{Y} \times\{1\})$. Since both maps are $Z$-imbeddings, there is a homeomorphism $\gamma_{3}: \bar{Y} \rightarrow \bar{Y}$ such that $\gamma_{3} \circ \gamma_{1}\left|(\bar{X} \times\{0\} \cup \bar{Y} \times\{1\})=\gamma_{2}\right|(\bar{X}$ $\times\{0\} \cup \bar{Y} \times\{1\})$. Thus, $\gamma_{2}^{-1} \circ \gamma_{3} \circ \gamma_{1}: M(\bar{f}) \rightarrow M(\bar{g})$ is the desired homeomorphism.

Proposition 3.3. Let $X, Y$, and $Z$ be locally compact $A N R s$ and let $f$ : $X \rightarrow Y, g: Y \rightarrow Z$ be proper maps. Then:

(i) There are proper homotopy equivalences $H$ and $G$ between $M(g \circ f)$ and $M(f, g)$ which are the identity on the ends and such that the homotopies from $G \circ H$ and $H \circ G$ to id are the identity on the ends at each level.

(ii) $M(\bar{g} \circ \bar{f})$ is homeomorphic to $M(\bar{f}, \bar{g})$ via a homeomorphism which is the identity on the ends.

Proof. The proof is similar to the proof of Proposition 3.2. (ii) $\Rightarrow$ (i) and (ii) is proven by projecting to the base and unknotting.

Proposition 3.4. Let $X$ and $Y$ be locally compact $A N R$ s and let $f, g: X \rightarrow Y$ be proper homotopic maps. Then

(i) $T(f)$ is proper homotopy equivalent to $T(g)$.

(ii) $T(\bar{f})$ is homeomorphic to $T(\bar{g})$.

Proof. These follow directly from Proposition 3.2.

REMark. Part (i) of each proposition can be proven in greater generality by other methods. We use infinite dimensional methods because they are convenient in our context.

4. Constructing approximate fibrations. Let $\mathfrak{p}: \mathcal{E} \rightarrow S^{1}$ be a fibration with $\mathcal{E}$ an ANR and let $\mathscr{F}=\mathfrak{p}^{-1}(1)$ be homotopy dominated by a finite CW 
complex. Represent $S^{1}$ by $S^{1}=\left\{e^{2 \pi i t} \mid 0<t<1\right\}$. By the covering homotopy property, there is a map $\varphi: \mathscr{F} \times I \rightarrow \mathcal{E}$ such that $\varphi_{0}=$ inclusion and $\mathfrak{p} \circ \varphi_{t}=$ $e^{2 \pi i t}$. The map $\varphi_{1}: \mathscr{F} \rightarrow \mathscr{F}$ is called the characteristic homotopy equivalence. It is well defined up to homotopy. Since $\mathscr{F}$ is an ANR, $\mathscr{F}$ has the homotopy type of a locally finite CW complex $L$. Let $w: \mathscr{F} \rightarrow L$ be a homotopy equivalence and let $v: L \rightarrow \mathscr{F}$ be a homotopy inverse for $w$.

The map $\varphi_{2}=w \varphi_{1} v: L \rightarrow L$ is a homotopy equivalence. By Proposition 2.4, $\overline{\bar{\varphi}}_{2}: \overline{\bar{L}} \rightarrow \overline{\bar{L}}$ is homotopic to a homeomorphism $\varphi_{3}: \bar{E} \rightarrow \bar{L}$. The natural projection $p: T\left(\varphi_{3}\right) \rightarrow S^{1}$ is a locally trivial bundle map. It is not difficult to construct a fiber homotopy equivalence from $\left(\mathcal{E}, \mathfrak{p}, S^{1}\right)$ to $\left(T\left(\varphi_{3}\right), p, S^{1}\right)$.

Proposition 4.1. Let

$$
K \stackrel{d}{\stackrel{\vec{u}}{\rightleftharpoons}} L
$$

be a homotopy domination with $K$ a finite complex and let $\Phi=u \varphi_{2} d: K \rightarrow K$. Then there is a homotopy equivalence between $T(\Phi)$ and $T\left(\varphi_{3}\right)$ such that the diagram

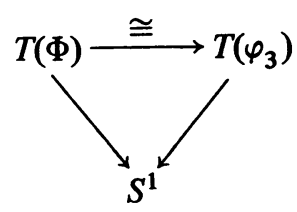

homotopy commutes.

Proof. By Proposition 3.4 we have homotopy equivalences $T(\Phi) \simeq T(\overline{\bar{\Phi}})$ $=T\left(\overline{\bar{u}} \overline{\bar{p}}_{2} \bar{d}\right) \simeq T\left(\overline{\bar{u}} \bar{\varphi}_{3} \overline{\bar{d}}\right)$ which homotopy commute with the natural projections to $S^{1}$. By Proposition 2.5, the map $\overline{\bar{u}}: \overline{\bar{L}} \rightarrow \overline{\bar{K}}$ is homotopic to an imbedding. If $\overline{\bar{L}}$ is identified with its image under this imbedding, $\overline{\bar{d}} \mid \overline{\bar{L}}: \overline{\bar{L}} \rightarrow \overline{\bar{L}}$ is homotopic to the identity. By the homotopy extension theorem (and Proposition 2.5), $\overline{\bar{d}}$ is homotopic to a proper retraction, $r: \overline{\bar{K}} \rightarrow \overline{\bar{L}}$.

Thus, $T\left(\overline{\bar{u}} \varphi_{3} \overline{\bar{d}}\right) \cong T\left(\varphi_{3} r\right) \cong T\left(r, \varphi_{3}\right)$. Note that $T\left(r, \varphi_{3}\right)$ contains a copy of $T\left(\varphi_{3}\right)$ (see Figure 2).

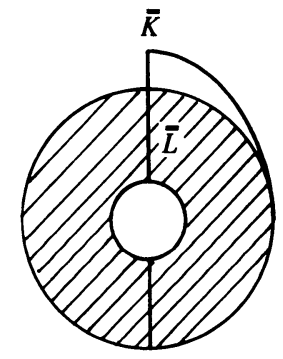

Figure 2

A rotation of $180^{\circ}$ clockwise carries all of $T\left(r, \varphi_{3}\right)$ into the shaded region. Since the shaded region is carried into itself, the $180^{\circ}$ rotation is homotopic to 
a deformation retraction of $T\left(r, \varphi_{3}\right)$ onto the shaded region. Thus, there is a homotopy equivalence $T\left(r, \varphi_{3}\right) \simeq T\left(\varphi_{3}\right)$.

Remark 4.2. Proposition 4.1 is based on an observation from [Fa]. The proof above illustrates the techniques we shall use to prove Proposition 4.3. A more pedestrian proof can be constructed using the Whitehead theorem.

We have shown that there is a homotopy equivalence making

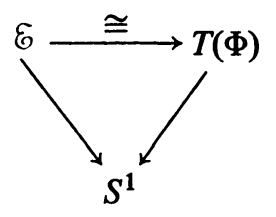

homotopy commute. The proof of Theorem 1.1 will be complete with the proof of the following.

Proposition 4.3. The natural map $T(\bar{\Phi}) \rightarrow S^{1}$ is homotopic to an approximate fibration.

Proof. Recall that $\Phi=u \varphi_{2} d$ where

$$
K \underset{u}{\stackrel{d}{\rightleftharpoons}} L
$$

is a homotopy domination and $\varphi_{2}: L \rightarrow L$ is a homotopy equivalence. Let $\alpha=u d: K \rightarrow K$. Note that $\alpha \circ \alpha \simeq \alpha$ and that $\alpha \circ \Phi \simeq \Phi \circ \alpha \simeq \Phi$.

The proof will consist of several steps.

Step I. Let $T_{n}=T(\bar{\Phi}, \bar{\alpha}, \bar{\alpha}, \ldots, \bar{\alpha})$ be the mapping torus of $\bar{\Phi}$ followed by $2^{n}-1$ copies of $\bar{\alpha}$ and let $p_{n}: T_{n} \rightarrow S^{1}$ be the projection. For each $n \geqslant 0$, there is a homeomorphism $h_{n}: T_{n} \rightarrow T_{n+1}$ such that $d\left(p_{n+1} \circ h_{n}, p_{n}\right)<$ $1 / 2^{n-1}$, distance in $S^{1}$ being measured by normalized arc length.

Proof (I). By Propositions 3.2 and $3.3, M(\bar{\Phi}) \cong M(\bar{\alpha} \circ \bar{\Phi}) \cong M(\bar{\Phi}, \bar{\alpha})$ and $M(\bar{\alpha}) \simeq M(\bar{\alpha} \circ \bar{\alpha}) \cong M(\bar{\alpha}, \bar{\alpha})$ via homeomorphisms which are the identity on the ends. Piecing these together yields the desired homeomorphism. $h_{1}$ is illustrated in Figure 3 below. This completes the proof of Step I.

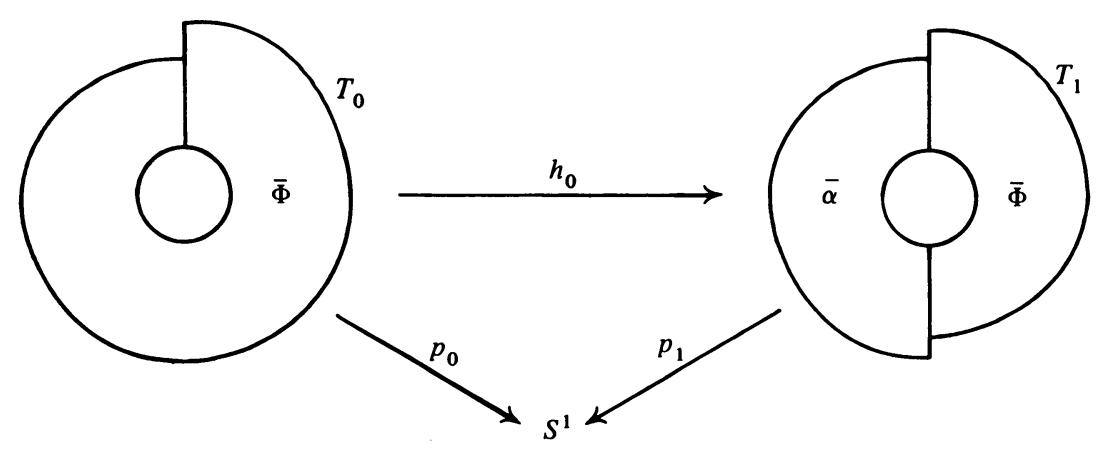

FIGURE 3 
Let $q_{n}=p_{n} \circ h_{n-1} \circ \ldots \circ h_{0}: T_{0}=T(\bar{\Phi}) \rightarrow S^{1}$. Then $d\left(q_{n}, q_{n+1}\right)=$ $d\left(p_{n+1} \circ h_{n}, p_{n}\right)<1 / 2^{n-1}$ and $q=\lim _{n \rightarrow \infty} q_{n}$ exists. Our plan is to show that $q$ is an approximate fibration by showing that each $q_{n}$ is a $\left(1 / 2^{n-3}\right)$-fibration. The result then follows as in Proposition 1.1 of $\left[C-D_{1}\right]$ where it is shown that the uniform limit of fibrations is an approximate fibration.

Step II. $q_{n}$ is a $\left(1 / 2^{n-3}\right)$-fibration if $p_{n}$ is.

Proof (II). Suppose that $p_{n}$ is a $\left(1 / 2^{n-3}\right)$-fibration and let $f: X \times I \rightarrow S^{1}$ and $F: X \times\{0\} \rightarrow T_{0}$ be given with $q_{n} \circ F=f \mid X \times\{0\} . q_{n} \circ F=$ $p_{n} \circ\left(h_{n-1} \circ \cdots \circ h_{0} \circ F\right)$, so, by hypothesis, there is a map $F^{\prime}: X \times I \rightarrow$ $T_{n}$ such that $d\left(p_{n} \circ F^{\prime}, f\right)<1 / 2^{n-3}$ and $F^{\prime}(x, 0)=h_{n-1} \circ \cdots \circ h_{0} \circ F$. Let $F^{\prime \prime}=\left(h_{n-1} \circ \cdots \circ h_{0}\right)^{-1} \circ F^{\prime} . F^{\prime \prime}$ is the desired lifting of $f$.

Step III. To show that $p_{n}: T_{n} \rightarrow S^{1}$ is a $\left(1 / 2^{n-3}\right)$-fibration, it suffices to find a map $\psi: T_{n} \times R^{\prime} \rightarrow T_{n}$ such that $\psi(x, 0)=x$ and $d\left(p_{n} \psi(x, t), p_{n}(x)+t\right)<$ $1 / 2^{n-3}$. Addition of $z \in S^{1}$ and $t \in R^{\prime}$ is performed modulo 1 and $z+t \in$ $S^{1}$.

Proof (III). Let $f: X \times I \rightarrow S^{1}$ and $F: X \times\{0\} \rightarrow T_{n}$ with $p_{n} \circ F=f \mid X$ $\times\{0\}$ be given and let $\tilde{f}: X \times I \rightarrow R^{1}$ be a lifting of $f(x, t)-f(x, 0)$ to the universal cover of $S^{1}$ such that $\tilde{f}(x, 0)=0$. Define $F^{\prime}: X \times I \rightarrow T_{n}$ by $F^{\prime}(x, t)=\psi(F(x, 0), \tilde{f}(x, t)) . F^{\prime}(x, 0)=\psi(F(x, 0), 0)=F(x, 0)$ and

$$
\begin{aligned}
d\left(p_{n} \circ F^{\prime}(x, t)\right) & =d\left(p_{n} \circ \psi(F(x, 0), \tilde{f}(x, t)), p_{n} \circ F(x, 0)+\tilde{f}(x, t)\right) \\
& <1 / 2^{n-3} .
\end{aligned}
$$

Step IV. The construction of $\psi$.

Proof (IV). To construct $\psi: T_{n} \times R^{1} \rightarrow T_{n}$ it suffices to construct $\psi_{1}$ : $\overline{\bar{T}}_{n} \times R^{1} \rightarrow \overline{\bar{T}}_{n}$ such that $d\left(\bar{p}_{n} \psi_{1}(x, t), \bar{p}_{n}(x)+t\right)<1 / 2^{n-3}$. If $\psi_{1}$ is such a function then the composition

$$
T_{n} \times R^{1} \stackrel{\times 0}{\rightarrow} T_{n} \times R^{1} \stackrel{\psi_{1}}{\rightarrow} T_{n} \stackrel{\text { proj }}{\rightarrow} T_{n}
$$

is the desired function $\psi$.

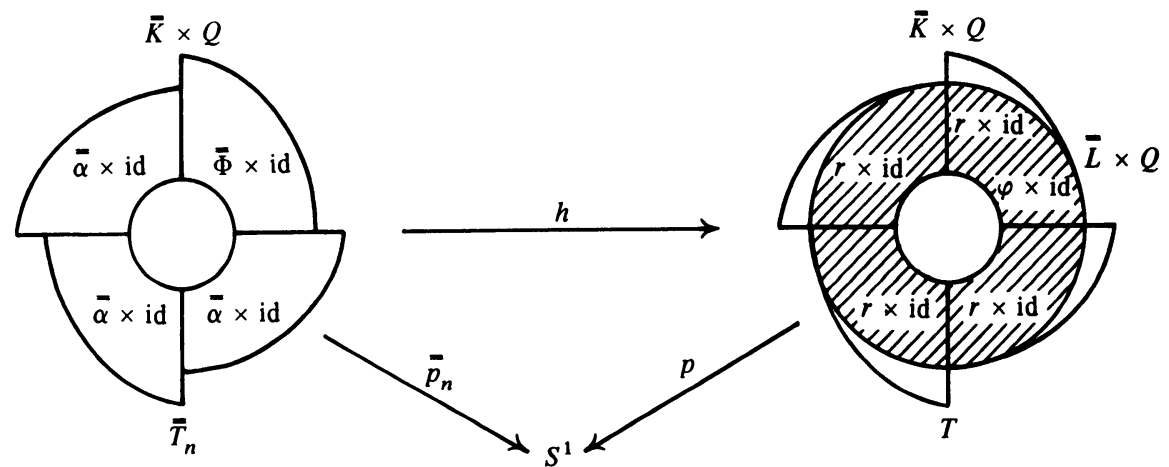

FIGURE 4 
$T_{n} \times[0,1)=T(\overline{\bar{\Phi}}, \overline{\bar{\alpha}}, \ldots, \overline{\bar{\alpha}})$. As in the proof of Proposition 4.1, $\overline{\bar{\alpha}}$ is homotopic to a retraction $r: \overline{\bar{K}} \rightarrow \overline{\bar{L}} \subseteq \overline{\bar{K}}$ and $\overline{\bar{\Phi}}$ is homotopic to the composition of $r$ with a homeomorphism $\varphi: \overline{\bar{L}} \rightarrow \overline{\bar{L}}$. By Propositions 3.2 and 3.3 there is a homeomorphism $h: T(\overline{\bar{\Phi}}, \overline{\bar{\alpha}}, \ldots, \overline{\bar{\alpha}}) \times Q \rightarrow T$ where $T$ is the space pictured in Figure 4. Note that $T(\overline{\bar{\Phi}}, \overline{\bar{\alpha}}, \ldots, \overline{\bar{\alpha}}) \times Q=\overline{\bar{T}}_{n}$. $h$ can be chosen so that $d\left(p \circ h, \overline{\bar{p}}_{n}\right)<1 / 2^{n}$, where $p: T \rightarrow S^{1}$ and (by abuse of our notation) $\overline{\bar{p}}_{n}: \overline{\bar{T}}_{n} \rightarrow S^{1}$ are the projections.

The shaded region in Figure 4 is a fiber bundle with fiber $\overline{\bar{L}} \times Q$ and characteristic homeomorphism $\varphi \times$ id. We describe a map $\psi_{2}: T \times R^{1} \rightarrow T$.

For $0<|t| \leqslant 1 / 2^{n}, \psi_{2}$ rotates $T$ one notch to the right. This carries $T$ down the mapping cylinders and into the shaded region. For $|t| \geqslant 1 / 2^{n}, \psi_{2}$ is a map into the shaded region which covers the map $f: T \times R^{1} \rightarrow S^{1}$ defined by

$$
f(x, t)= \begin{cases}t, & t \geqslant 1 / 2^{n}, \\ t+1 / 2^{n-1}, & t \leqslant-1 / 2^{n} .\end{cases}
$$

Thus, we have $d\left(p(x)+t, p \circ \psi_{2}(x, t)\right) \leqslant 1 / 2^{n-1}$.

Let $\psi_{1}=h^{-1} \circ \psi_{2} \circ(h \times$ id $)$. Then

$$
\begin{gathered}
d\left(p_{n}(x)+t, p_{n} \circ \psi_{1}(x, t)\right)=d\left(p_{n}(x)+t, p_{n} \circ h^{-1} \circ \psi_{2}(h(x), t)\right) \\
<d\left(p_{n}(x)+t, p \circ h(x)+t\right)+d\left(p \circ h(x)+t, p \circ \psi_{2}(h(x), t)\right) \\
\quad+d\left(p \circ \psi_{2}(h(x), t), p_{n} \circ h^{-1} \circ \psi_{2}(h(x), t)\right) \\
<1 / 2^{n}+1 / 2^{n-1}+1 / 2^{n}=1 / 2^{n-2}<1 / 2^{n-3} .
\end{gathered}
$$

This completes the proof of Step IV, Proposition 4.3, and Theorem 1.1.

\section{REFERENCES}

[A-Ch] R. D. Anderson and T. A. Chapman, Extending homeomorphisms to Hilbert cube manifolds, Pacific J. Math. 38 (1971), 281-293. MR 47 \#7749.

[B] K. Borsuk, Theory of shape, Lecture Notes Ser., no. 28, Matematisk Institut, Aarhus Univ., Aarhus, 1971. MR 45 \# 2679.

[Ch] T. A. Chapman, Lectures on Hilbert cube manifolds, CBMS Regional Conf. Ser. in Math., Amer. Math. Soc., Providence, R.I. (to appear).

[Ch-F $]$ T. A. Chapman and S. Ferry, Obstruction to finiteness in the proper category (preprint).

[Ch-F $\left.\mathrm{F}_{2}\right]$, Hurewicz fiberings with ANR fibers, Topology (to appear).

[C] M. Cohen, A course in simple-homotopy theory, Springer-Verlag, Berlin and New York, 1973.

[C-D, D. S. Coram and P. F. Duvall, Approximate fibrations (preprint).

$\left[\mathrm{C}-\mathrm{D}_{2}\right] \ldots$, Approximate fibrations and a movability condition for maps (preprint).

[E-G] D. A. Edwards and R. Geoghegan, Shapes of complexes, ends of manifolds, homotopy limits, and the Wall obstruction, Ann. of Math. (2) 101 (1975), 521-535. MR 51 \#11525.

[Fa] F. T. Farrell, The obstruction to fibering a manifold over a circle, Actes Congres Internat. Math. (Nice, 1970), Tome 2, Gauthier-Villars, Paris, 1971, pp. 69-72.

[G] R. E. Goad, Local homotopy properties of maps and approximation by fiber bundle projection, Thesis, Univ. of Georgia, 1976. 
[H] L. S. Husch, Approximating approximate fibrations by fibrations. I, II (preprint).

[L] R. C. Lacher, Cell-like mappings. I, Pacific J. Math. 30 (1969), 717-731. MR 404941.

[M] L. Mand, A generalization of cell-like mappings, Thesis, Univ. of Kentucky, 1975.

[S-G-H] L. Siebenmann, L. Guillou, and H. Hahl, Les voisinages ouverts réguliers: critères homotopiques d'existence, Ann. Sci. Ecole Norm. Sup. (4) 7 (1974), 431-462 (1975). MR 50 \#14766.

[W] J. E. West, Mapping Hilbert cube manifolds to ANR's, Ann. of Math. (to appear).

$\left[\mathrm{W}_{2}\right]$, Mapping cylinders of Hilbert cube factors. II, General Topology and Appl. 1 (1971), no. 2, 111-125. MR 44 \#5984.

Department of Mathematics, University of Kentucky, Lexinton, Kentucky 40506 